\title{
Design and Operation of Coherent Optical Radar for Civilian Applications
}

\author{
Dr. Mahdi S. Edan \\ Department of Quality Assurance, Ministry of Higher Education and Scientific Research, \\ Baghdad, Iraq \\ Northern Technical University - Mosul \\ mafcd@mst.edu, mahdiedan@yahoo.com
}

\begin{abstract}
This paper introduces experimental results of a laboratory scale laser Radar. A computer-controlled laser radar system for measuring moving object velocities using both $\mathrm{CO}_{2}$ laser and laser diode was constructed. Different moving objects with different velocities were used. Furthermore, Doppler Shifts, modulation frequencies and laser wavelengths were transferred to a personal computer (PC). From the previous parameters, the pc was able to present target velocities. A special software and the relevant electronic card (type HP-Gp-lb-designed for spectrum analyzer) were used for noise cancellation and for measuring moving object velocities. We used heterodyne detection technique to detect weak signals.
\end{abstract}

Keywords: Laser; Laser radar; Heterodyne detection; Coherent optical radar 


\section{Introduction}

Impressive advances in using laser radiation in Ladar (Laser-Detection and Ranging), have been demonstrated in several research laboratories and in field applications. Heterodyne detection techniques play a crucial role in building coherent radar systems. Their receivers have significant importance in the scientific exploration of the earth's atmosphere and in the investigation of astronomical objects [1]. The high spectral resolution of heterodyne receivers is used from the microwave $(\lambda=10 \mathrm{~mm})$ to the thermal infrared $(\lambda=10.6 \mu \mathrm{m})$, to derive the volume mixing ratio profiles of stratospheric trace gases, to determine absolute and relative abundance of chemical species in the interstellar medium, and to deduce the dynamical information from the fine structure of the spectra [2]. The basic heterodyne principle depends on mixing two beams to achieve the beating frequency, which impinges on the photos-detector surface. The heterodyne optical receiver uses a laser called the Local oscillator laser, together with a photodiode, to convert an incoming optical signal into an electrical signal called the intermediate frequency (IF) signal [3]. The local oscillator power which proves to have great effect on increasing the signal from target comes from the same laser source. It will in turn facilities the tuning processes and signal reducing the associated noise [4].

Optical communication systems that use a local oscillator are frequently called coherent systems. Coherent optical radar offers nearly ideal characteristics for several reasons. First, the heterodyne receiver behaves as a nearly ideal optical filter [5]. The receiver discriminates against any noise outside the required signal bandwidths. This can be achieved using electrical band bass filters. They have broad band widths ranging from few MHZ to a few GHZ. Furthermore, they have sharp edges and flat responses [6].

The second advantage is that coherent transmitters use single - frequency laser source with line widths typical in the range of 1 to $25 \mathrm{MHZ}$ [7]. When these sources are modulated at speeds of $100 \mathrm{Mb} / \mathrm{s}$ to $10 \mathrm{~Gb} / \mathrm{s}$, the resulting optical spectral width is comparable to the information bandwidth of the modulating signal. The narrow spectral width reduces the influence of chromatic dispersion in the fiber to a minimum [8]. Further advantage is weak laser signals which are reflected from a target can be easily detected using heterodyne detection technique. It enforces the returned signal from target and facilitate measuring Doppler shift (DF) [9].

DF arises due to the variation in the returned laser frequency from a moving object [10]. It is used to measure fluids, moving objects velocity and to calculate the number of polluting particles in a moving fluid. Signal to noise ratio $(\mathrm{S} / \mathrm{R})$ is very much higher in the coherent detection as compared to direct detection (see Fig. 1). 
The heterodyne technique can also be applied to pulsed signals by using a pulsed local oscillator beam. It is even possible to use ultrashort pulses. As the heterodyne detector is essentially sensitive only while the local oscillator light is there (assuming a signal power well below the local oscillator power), it is possible to obtain a very high temporal resolution without requiring a very fast photodetector. This technique is called heterodyne (or homodyne) linear optical sampling [11,12].

According to the numerical results, the variation of displacement resolution against the key parameters including the laser wavelength, the detection distance, the laser linewidth and the signal power. It reveals that the phase fluctuation has a significant effect on the power spectrum of photocurrent [13]. Besides, both the probability density distribution of phase noise and displacement resolution have heavy dependence on laser oscillation line shapes. And the noiseequivalent mean square displacement is inversely proportional to the root-mean-square of a measurable light power [14].

\section{Experimental}

The Experimental setup which was used to study the coherent detection is shown in figure (2). A sealed - off waveguide $\mathrm{CO}_{2}$ laser with the following specifications was used:

The $\mathrm{CO}_{2}$ Waveguide Laser Source Specifications:

Output $=4$-watt Mode structure: single mode

Beam diameter : $1.3 \mathrm{~mm}$ at e points

Beam divergence: $10 \mathrm{mrad}$, full angle

Cooling Requirement: $0.25 \mathrm{gal} / \mathrm{min}$

Further instruments were liquid nitrogen cooled Mercury Cadmium Telluride (M.C.T) photoconductive detector, expanding and collimating Galilean telescope, and 3 pieces of germanium windows with diameters of $3 \mathrm{~cm}$ each and mirrors of 5-7.5 $\mathrm{cm}$ diameters were used (7). The CO2 laser was replaced in some experiments with a semi- conductor laser $(\lambda=810$ $\mathrm{nm}$ ) and silicon detector. An acoustic optic modulator, RF power amplifier with analogue driver were used to modulate the output laser beam.

A spectrum analyzer having 0-22 GHz frequency band was used to measure Doppler shifts and to know the direction of moving objects. It could measure powers as low as $1130 \mathrm{dBm}$.

\section{Spatial filtering}

The Influence of band pass filter on the interference and on achieving the coherent detection is also studied. It may passively or negativity affects interference. This is decided by the effective area of detector. In heterodyne microwaves receivers, band pass filters determine the required frequency band to pass to the receiver. Consequently, it determines the beating frequency band and eliminates a great amount of noise. In our current research study, we used 
a spatial filter (pinhole) with a varying hole diameter from $0.5-1.5 \mathrm{~mm}$. The effect of pinhole size on signal amplitude is to reach the optimum coherent detection efficiency at $1.3 \mathrm{~mm}$. However, at $0.7 \mathrm{~mm}$, the gain was significantly reduced as well as it was difficult to align the system (see figure3). Other parameters affecting coherent detection namely, atmospheric attenuation was studied. $\mathrm{CO} 2$ laser transmission was found to reduce to $87 \%$ at $10 \%$ humidity and to $4 \%$ at $90 \%$ humidity. We observed that coherent detection helps in detecting signals even when humidity increases to $90 \%$. The signal gain was $6 \mathrm{~dB}$ at coherent detection in comparison to $1 \mathrm{~dB}$ at direct detection.

The type of reflecting object effect is also studied. As an example, the signal amplitude was $60 \mathrm{~dB}$ using gold surface. This number was reduced to $35 \mathrm{~dB}$ and $30 \mathrm{~dB}$ using aluminum and germanium respectively. Furthermore, beating signal was found to increase when increasing the local oscillator power till 10 times then beating signal disappears and coherence is cancelled. The best modulation frequency was at $10 \mathrm{KHZ}$. The obtained signal and Doppler shift were very clear especially for slow moving objects.

When the reflected frequency from moving object is changed due to its velocity, then the signal on the spectrum analyzer will split and a second signal will be drawn to the right or to the left of the original signal (depending on object's direction). It represents frequency shift or Doppler shift which can be calculated as follows:

$$
\Delta \mathrm{v}=\frac{\mathrm{V} \cos \theta}{\lambda}
$$

where $\Delta v$ is the Doppler shift, $v$ moving target velocity, $\lambda$ laser radiation wavelength, and $\theta$ angle between the incident radiation on target and the direction of movement.

\section{Results and Discussions}

\section{A- Heterodyne Detection and Doppler shift:}

To prove the heterodyne detection principle, a Michelson - like set up using the $\mathrm{CO} 2$ laser system was constructed. This was to prove the heterodyne interference. The interference frequency which represents the interference between the LO wave and the reflected signal from a stationary target (S) is shown in figure (4). Doppler shift for a moving target towards the system with a velocity of $8.3 \mathrm{~mm} / \mathrm{sec}$ producing $1.58 \mathrm{KHz}$ Doppler shift is shown in figure (5). When blocking either the L.O or the reflected signal, the signal was found to disappear on the oscilloscope. This is further evidence for the occurrence of the H.D (i.e. Beating signal) from mixing both waves. When taking each wave alone, the wave gain was 35 $\mathrm{dB}$, while in mixing the gain was $40 \mathrm{~dB}$. This is justified due to the occurrence of beating frequency.

\section{$B$ - Laser Modulation}

Modulation of $100 \mathrm{~Hz}$ to $6 \mathrm{kHz}$ was achieved using the laser beam chopping technique as a simple modulation approach. Electronic modulation (i.e. interfacing an on-off electronic circuit to a power supply) was used to achieve $1 \mathrm{KHZ}$ to $10 \mathrm{KHZ}$ to the output laser radiation. Furthermore, an external modulator unit adapted to the $\mathrm{CO} 2$ laser was used to modulate the laser beam in the range $1 \mathrm{KHz}$ to $30 \mathrm{KHZ}$. Increasing modulation was found to decrease the laser power (see figure 6). This suggests that signal will disappear when using low laser powers at high degree of modulation. The best signal amplitude accompanied with clear Doppler shift was found to be at $10 \mathrm{KHZ}$ modulation. 
It is recommended that modulation should be twice as much as the Doppler shift. This guarantees no interference with Doppler shift on the spectrum analyzer screen and consequently recognizes target velocity.

\section{C- Computerizing the System}

The output signal from the radiation detector to the spectrum analyzer was processed in this work. This was to obtain several parameters needed for building the coherent laser radar. The aforementioned parameters were signal amplitude, modulation, Doppler shift and target speed and direction. HP- GP- IB electronic card to interface the spectrum analyzer to a personal computer was used. The objectives of using the card are to program the spectrum analyzer, determining the spectral range of the analyzer, sending information to the PC and simultaneously analyzing information including measuring target velocity. This was done through building a special software. The IEEE-488-GP-IB card is specially designed to communication between the spectrum analyzers (types 8566A\&8566B) for a maximum distance of 20 meter and the computer.

Four computer programs were produced to control the signal transformation process. The first is to program the analyzer through determining the working spectral range, bandwidth, gain $(\mathrm{dBm})$, and the sweep time. The second is to transform signal from analyzer to the pc, while the third program analyzes signal and types target velocity and Doppler shift values. It also shows the selection screen for the second time. Screen has the following selections: -

1- Save: It saves the signal.

2- Load: It loads a diagram from previous experiment.

3- End: to end and exit the program.

Quick basic language was used to write the programs. It was converted on a compact Disk (CD) as Execution program. These facilities used it even on personal computers having no quick basic.

Furthermore, a subroutine was added to eliminate noise and select Doppler shift only. Some pictures which were transformed from the analyzer using the pc are shown in figure (7). Target velocity and Doppler shift are shown on these figures. A program was designed to calculate Doppler shifts assuming three different laser wavelengths are shown in figure (8). It is shown that Doppler shift increases at the visible part of the electromagnetic spectrum in comparison to the infrared region. The experimentally obtained results are also shown in the figure for comparison.

\section{Conclusion}

In conclusion, a computer-controlled laser radar system for civilian applications was build and operated. It facilitated measuring velocities of moving targets using both $\mathrm{CO} 2(10.6 \mu \mathrm{m})$ and the semiconductor $(810 \mathrm{~nm})$ lasers. I also proved that the heterodyne detection technique reduced the noises and recommend using the sold state lasers in communications. In the laser applications the heterodyne detection technique is used to strengthen the signal and to measure the doppler frequency which caused by laser frequency reflected from moving target which was used for measurement of velocity of some liquids, to measure the impurities in liquid and amount of particles in moving liquids .

This technique also used in the military applications (radar and communications) to strengthen detection and reduce the noise signal. I demonstrated the heterodyne laser technique is double the value of the direct detection. 
Distribution of stimulated beat noise due to the reason of LO leads to reduce all other noise sources, therefore the $\mathrm{S} / \mathrm{N}$ ratio become unreliable on the level of power of the LO and theoretically where in:

$$
\mathrm{SNR}_{\mathrm{H}}=\frac{\eta \mathrm{P}_{\mathrm{S}}}{\mathrm{h} v \mathrm{~B}}
$$

$\eta$ : quantum efficiency for the detector

hv : photon energy

B: noise bandwidth

PS: power of the laser signal incident on the detector

The second important advantage for coherent direction lies in its ability to recognize the velocity of Doppler for targets through frequency shift for coherent signal which equals: $\mathrm{fD}=2 \mathrm{~V} / \lambda=200 \mathrm{KHz} / \mathrm{m} / \mathrm{s}$

when CO2 lasers use $(10.6 \mu \mathrm{m})$, it's best in applications of radar and range finders. 


\section{References}

1- B Partite, V Zeninari, C Thiebeaux, A Delahaigue, Review, infrared laser heterodyne systems, Spectrochemical Acta part A 602004 p1193-1213.

2- Richard E Wanger, Richard A Linke, Heterodyne light weight systems: Moving Towards Commercial Use, IEEE LCS Nov. 2005

3- Yas M R Al-Hadithi, Mahdi S Edan, M Al-Zuhairi, Mohammad S Mahdi, Heterodyne Detection Technique Using laser Radiation as a possible Technique for Remotely Senses the Wind Velocity, Environment Conference,June2004, Egypt, Cairo.

4- C.Dorrer eet al, "linear optical sampling", IEEE Photon Technol. Lett. 15 (12), 1746 (2003)

5- E. Benkler et al., "Characterization of ultrashot optical pulse properties by amp;itudemodulation -balanced heterodyne gating", Opt. Lett. 30 (15).2016 (2005).

6- YanChun-HuiabWangTing-FengaLiYuan-YangaLvTaoabWuShi-Songab,

"Investigating the displacement resolution of laser heterodyne detection system"optic Communications ,vol. 435, 15 march 2019,pages 68-74.

7- Yas M R Al-Hadithi, Mahdi S Edan, M Al-Zuhairi, Mohmmad S Mahdi, Laser Radiation Attenuation as a possible Approach for Estimating Humidity and pollution, Environment Conference, June 2004, Egypt, Cairo.

8- Copyright $\odot$, 1990-2001, The Cdumbus Optical SETI Observatory, http://www.coseti.org. 19/11/2000.

9- S. Rahman and D.A. Robertson, "Coherent $24 \mathrm{GHz}$ FMCW radar system for microDoppler studies", Proceedings Vol. 10633, Radar Sensor Technology XXII; 106330I (2018), SPIE Defense + Security, 2018, Orlando, Florida, United States.

10- F.Z. Zhang, Q.S. Guo and S.L. Pan, "Photonics-based real-time ultra-high-rangeresolution radar with broadband signal generation and processing", Scientific Reports, vol. 7, Article number: 13848 (2017).

11- P. S. Roy, M. D. Behera and S. K. Srivastav, "Satellite Remote Sensing: Sensors, Applications and Techniques", Proc. Natl. Acad. Sci., India, Sect. A Phys. Sci. (2017) 87: 465. https://doi.org/10.1007/s40010-017-0428-8.

12- M. Arend, M. Campmier, A. Fernandez and F. Moshary, "Application of Lidar and Other Profiling Techniques to Study the Impact That Severe Weather Hazards Have on the New York City Built Environment”, EPJ Web of Conference, 176, 06003, 2018, https://doi.org/10.1051/epjconf/201817606003

13- Yong Wang, Wenbin Ye, Yan Hong, Yuan Cao \& Yuanjin Zheng (2017) Feasibility study: highly integrated chipset design for compact synthetic aperture radar payload on micro-satellite, Journal of Electromagnetic Waves and Applications, 31:6, 594603, DOI: $10.1080 / 09205071.2017 .1298477$

14- M. Pieraccini, M. Fratini, F. Parrini, G. Macaluso and C. Atzeni, "High-speed CW stepfrequency coherent radar for dynamic monitoring of civil engineering structures," in Electronics Letters, vol. 40, no. 14, pp. 907-908, 8 July 2004. doi: 10.1049/el:20040549 


\section{COHERENT (HETERODYNE) VERSUS INCOHERENT SNR \\ As A Function of Background Ratio $P_{b} / P_{r}$ \\ And Electrical Output Bandwidth Be}

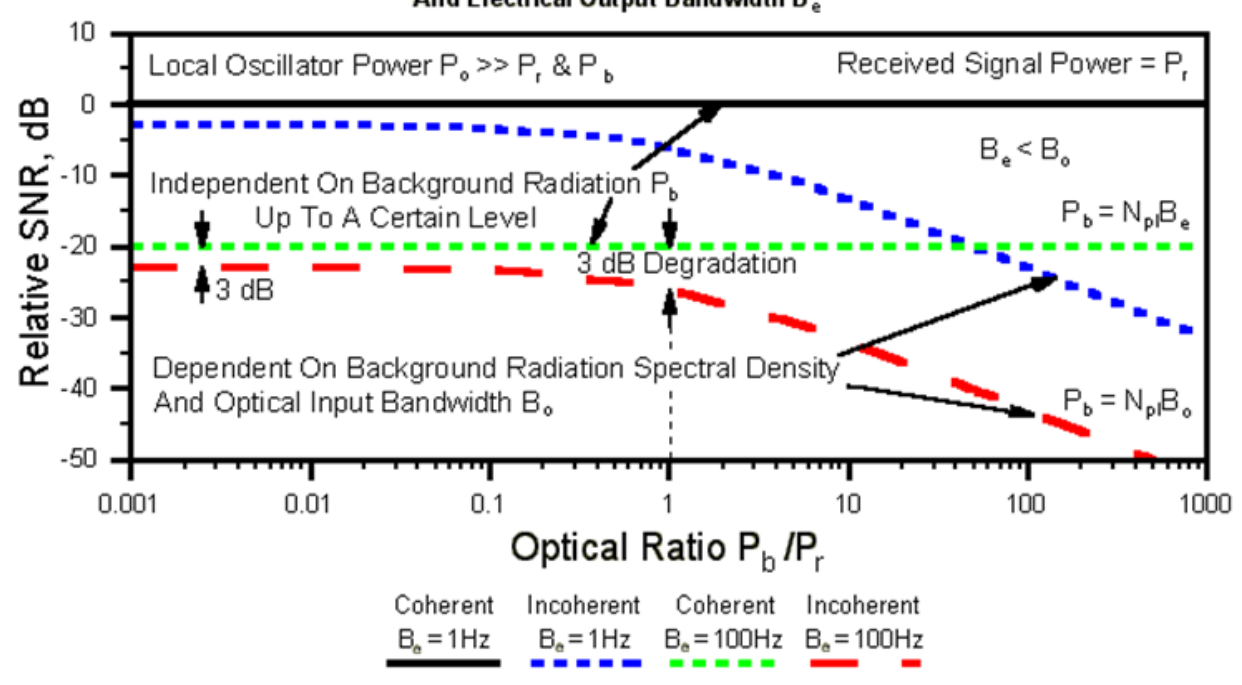

Fig. (1) Signal-to-Noise Ratio (SNR) using coherent and direct detection to received signal power

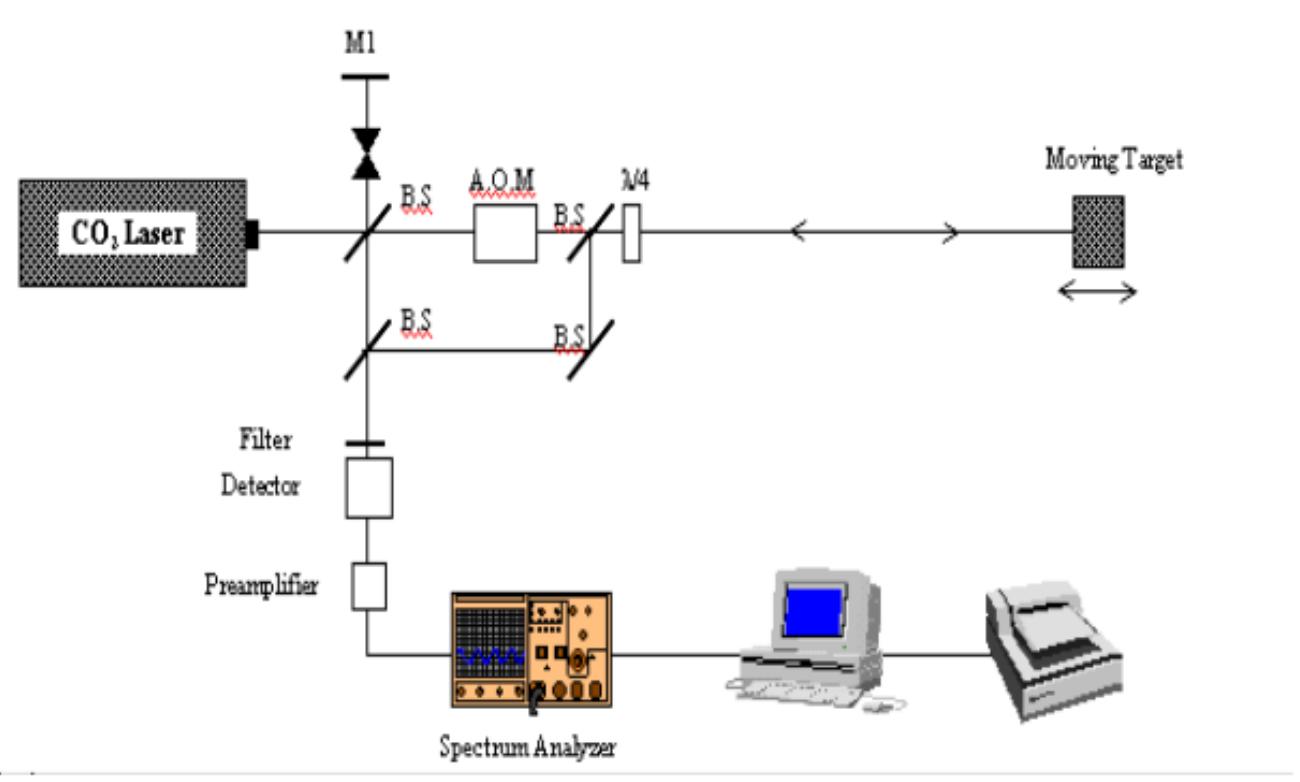

Fig. (2) Schematic showing Coherent detection system with the acousto-optic modulator 


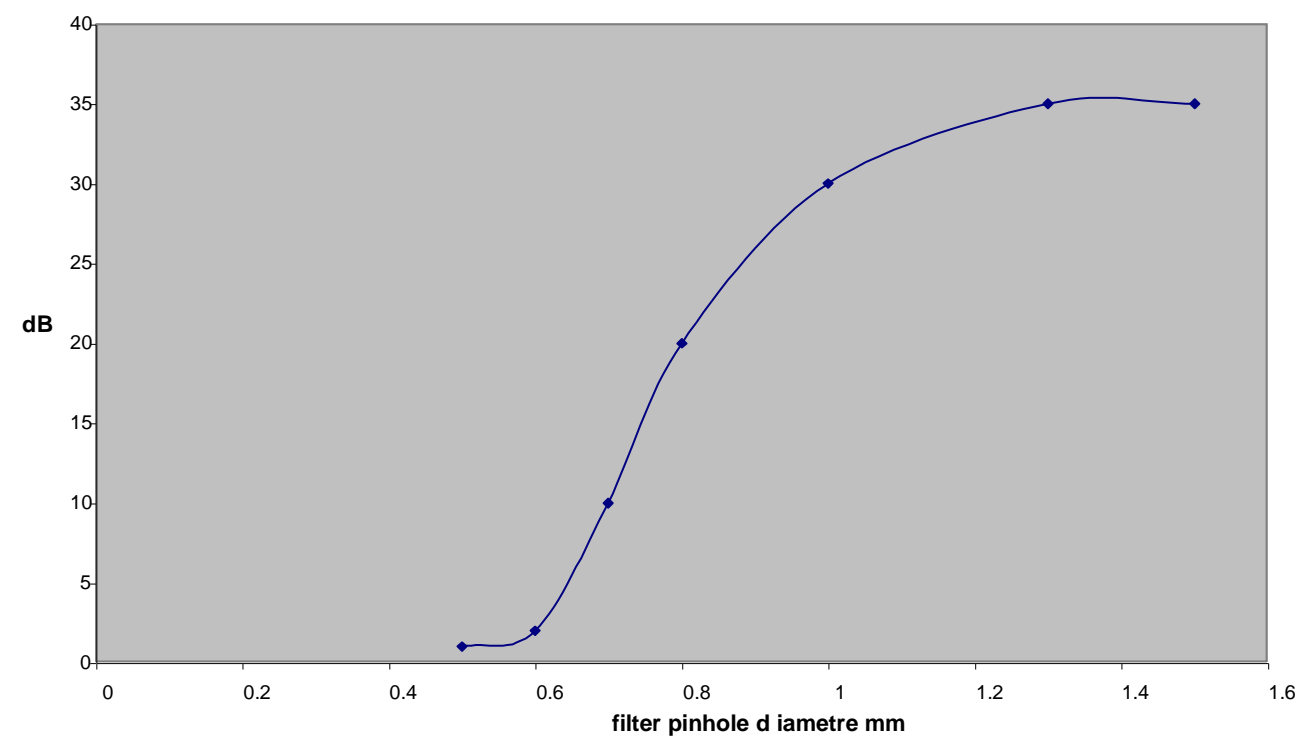

Fig. (3) The influence of spatial filter size on the signal amplitude experimentally observed

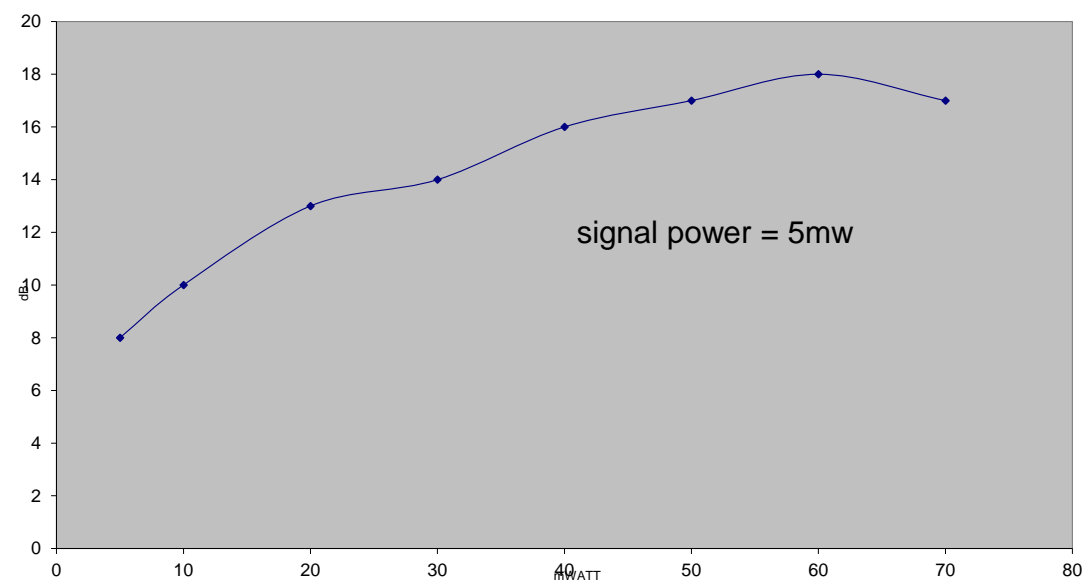



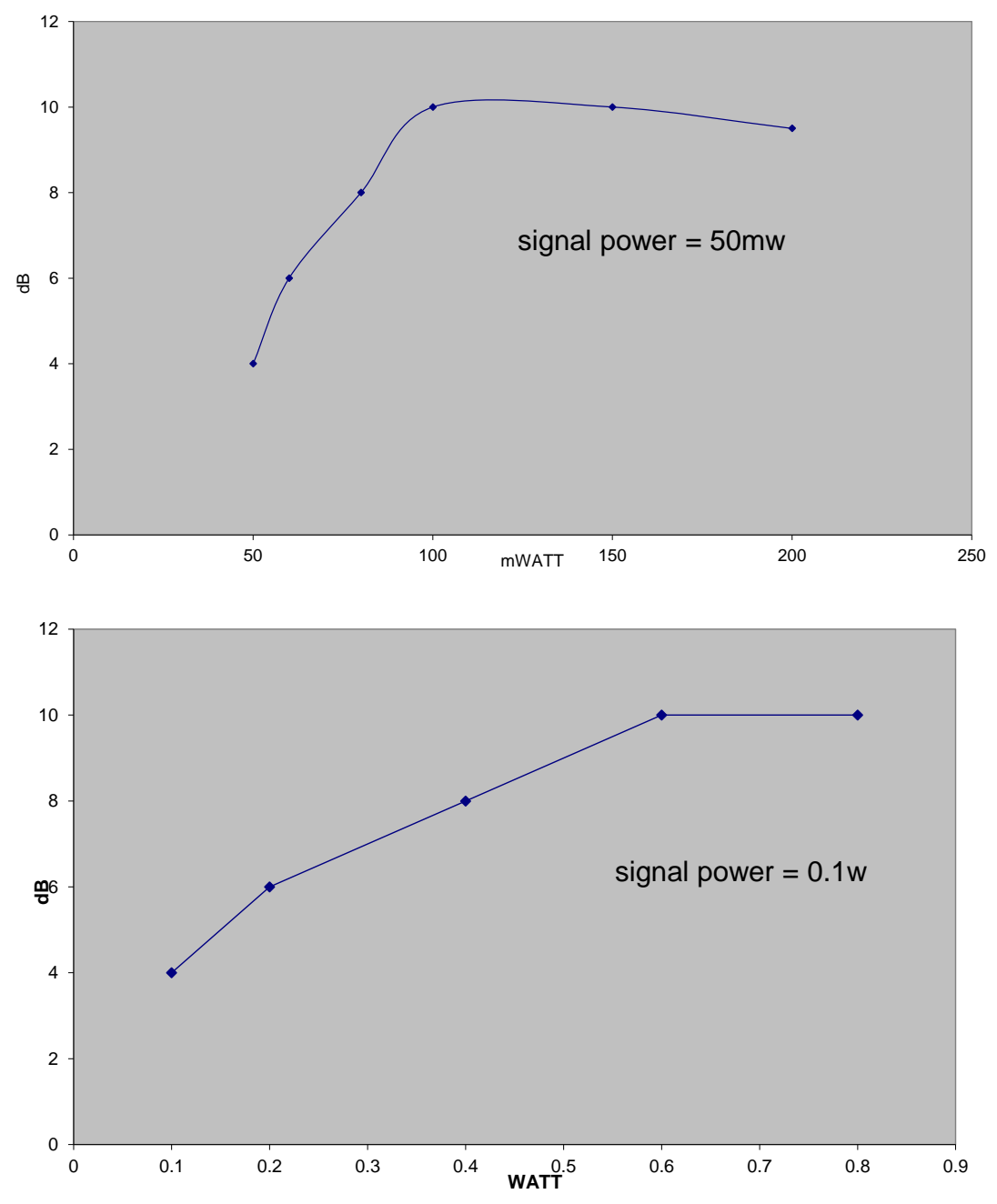

Fig. (4) The increase in the beating frequency with the increase of the local oscillator power 


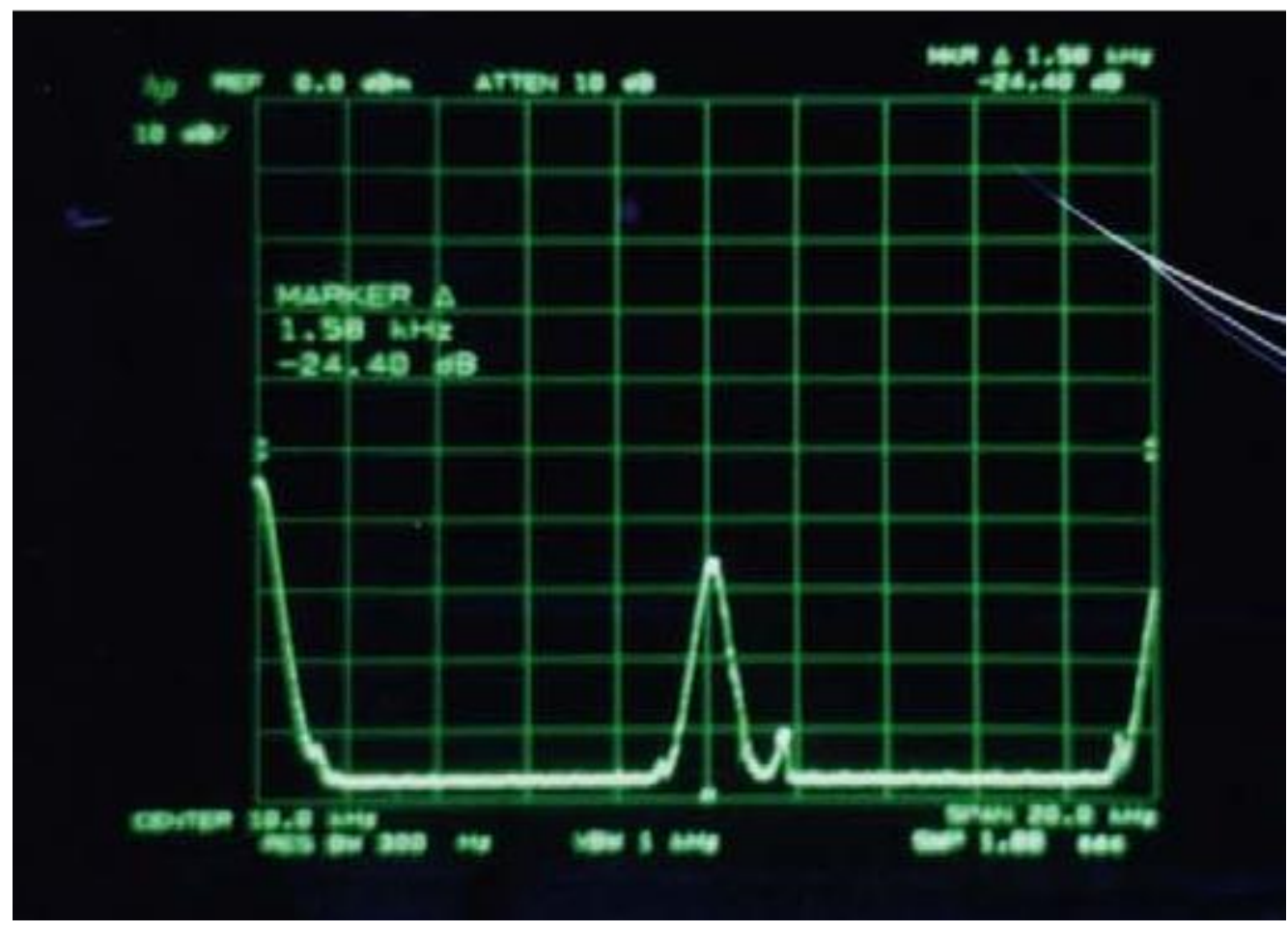

Fig. (5) Doppler shift for a moving target towards the system with a velocity of $8.3 \mathrm{~mm} / \mathrm{s}$ producing 1.58 kHz Doppler shift 


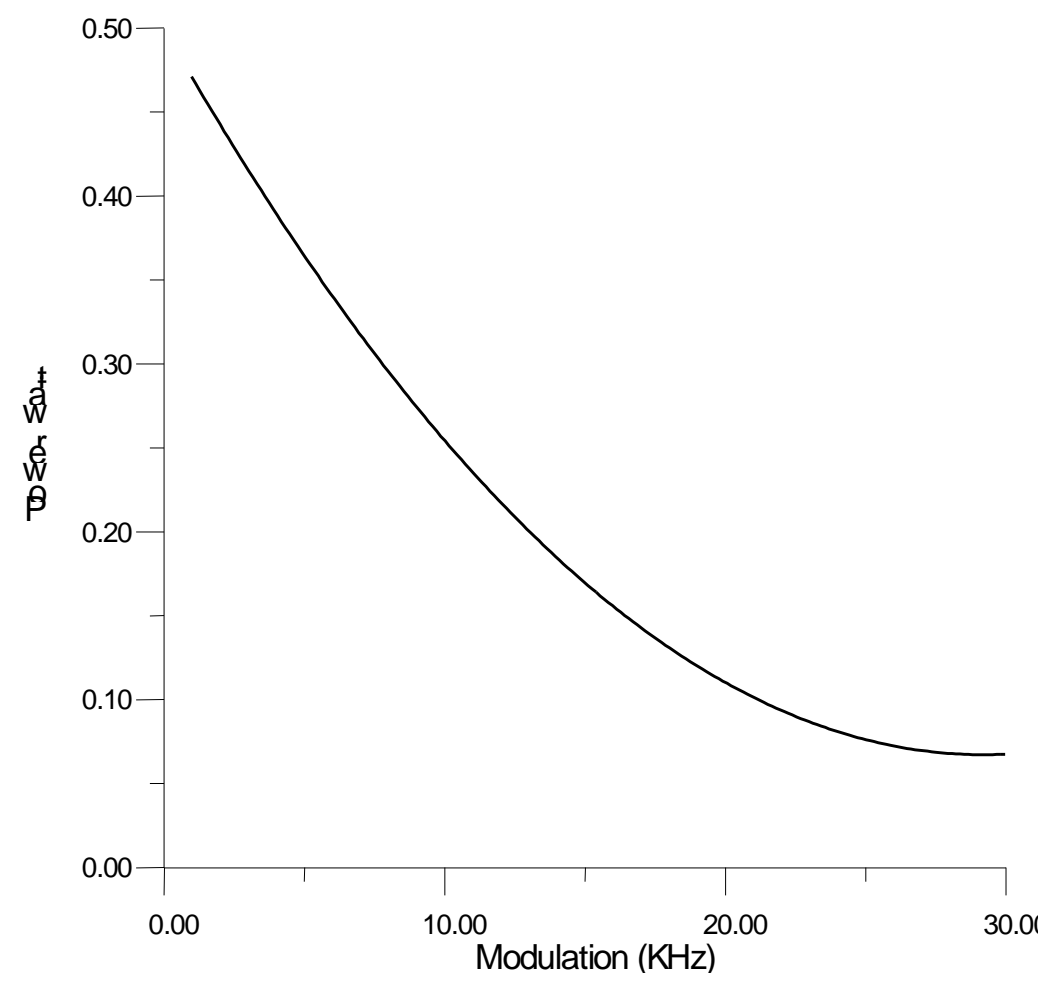

Fig. (6) The decrease of Laser power when increasing modulation

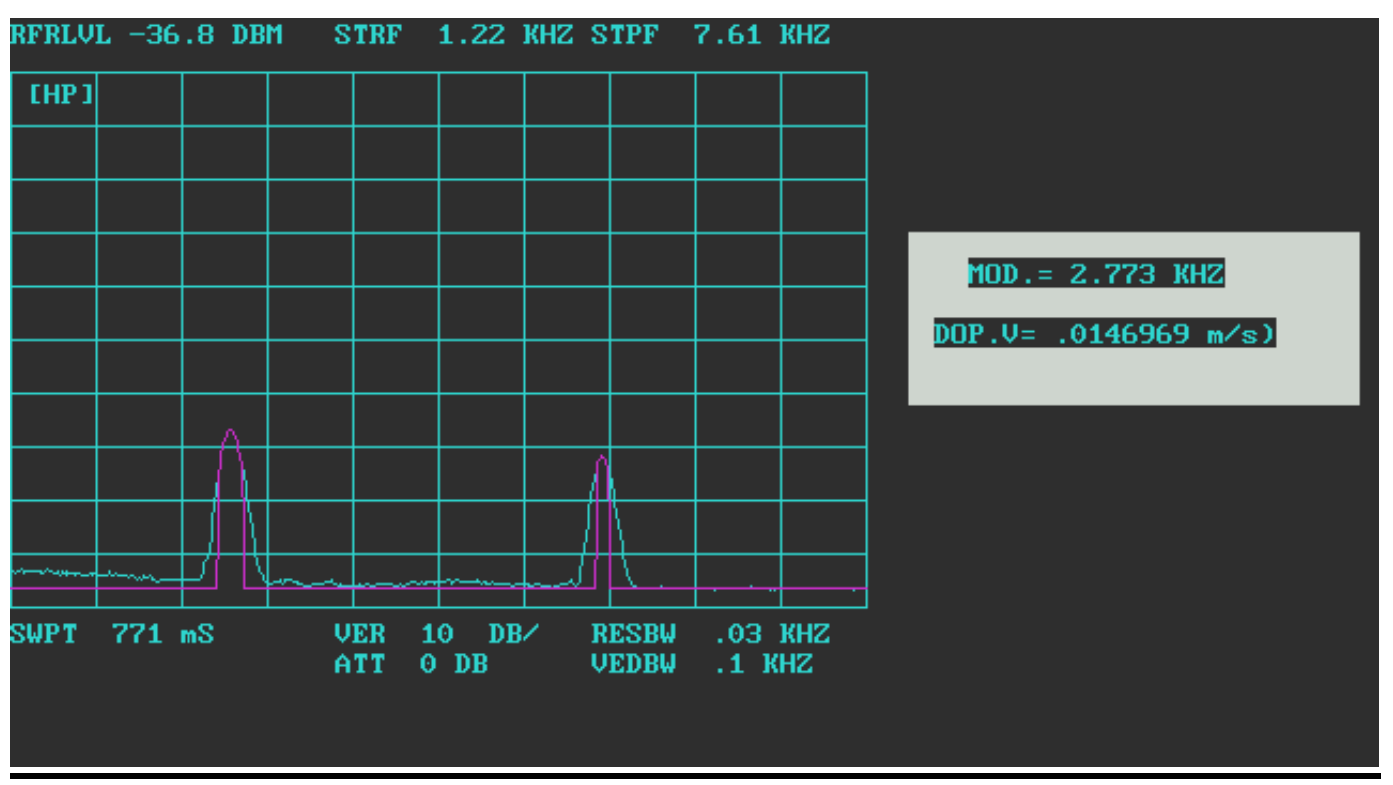




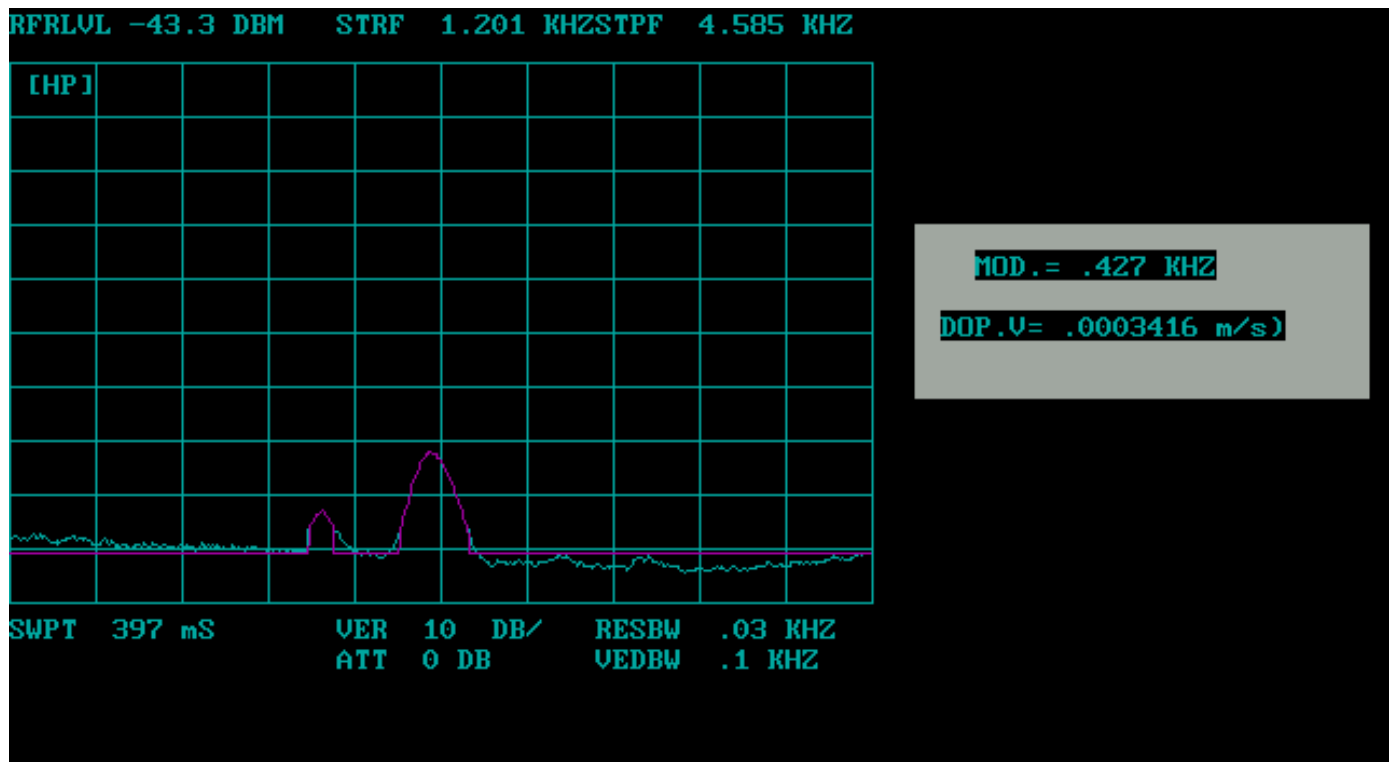

Fig. (7) Picture transformed from the spectrum analyzer using PC showing the Doppler velocity and the modulation frequency values 


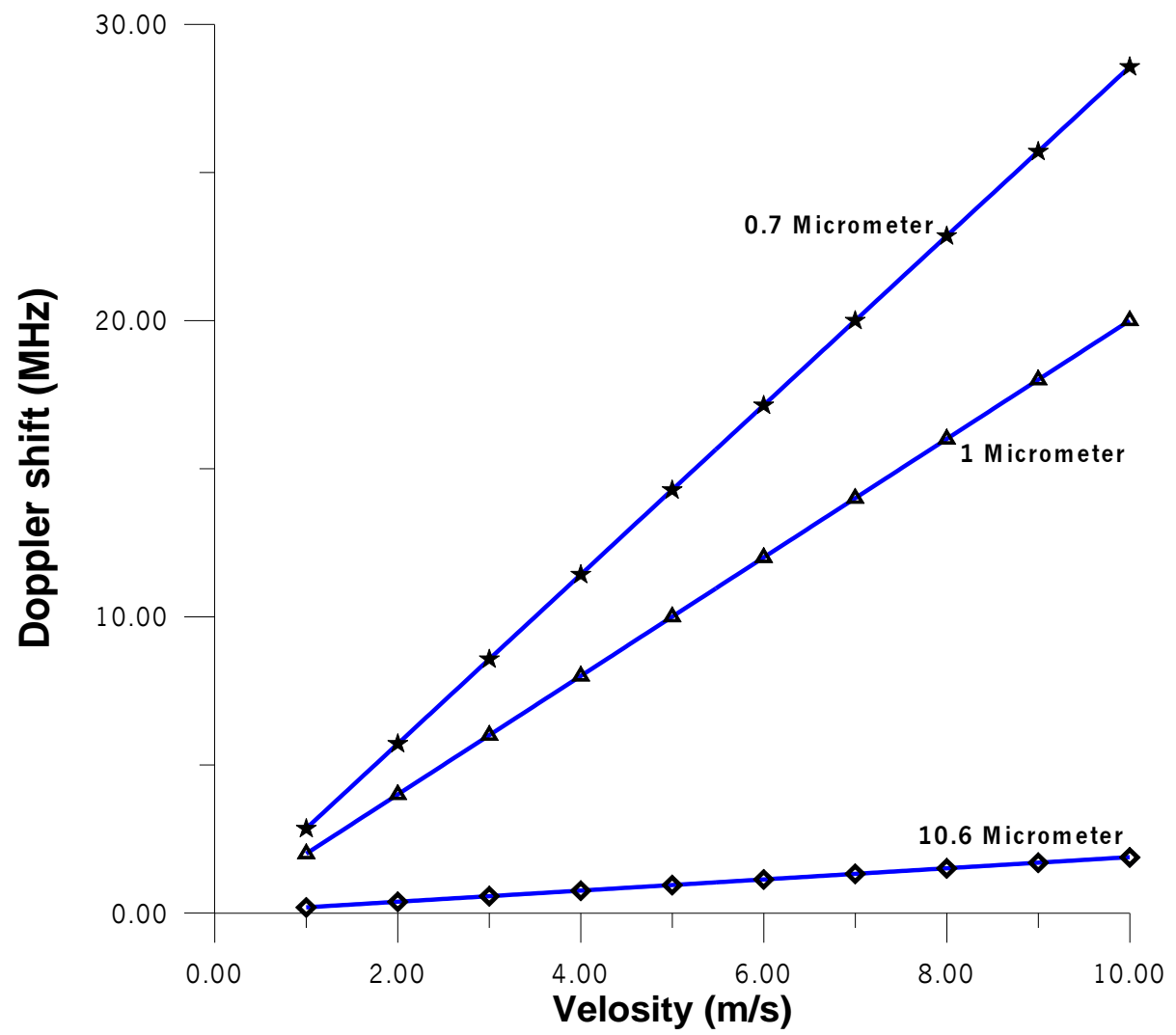

Fig. (8) Doppler shifts assuming three different laser wavelengths as a function of target velocity 